PROCEEDINGS OF THE

AMERICAN MATHEMATICAL SOCIETY

Volume 137, Number 9, September 2009, Pages 2921-2934

S 0002-9939(09)09883-9

Article electronically published on April 2, 2009

\title{
CSP DICHOTOMY FOR SPECIAL TRIADS
}

\author{
LIBOR BARTO, MARCIN KOZIK, MIKLÓS MARÓTI, AND TODD NIVEN
}

(Communicated by Jim Haglund)

\begin{abstract}
For a fixed digraph $\mathbb{G}$, the Constraint Satisfaction Problem with the template $\mathbb{G}$, or $\operatorname{CSP}(\mathbb{G})$ for short, is the problem of deciding whether a given input digraph $\mathbb{H}$ admits a homomorphism to $\mathbb{G}$. The dichotomy conjecture of Feder and Vardi states that $\operatorname{CSP}(\mathbb{G})$, for any choice of $\mathbb{G}$, is solvable in polynomial time or NP-complete. This paper confirms the conjecture for a class of oriented trees called special triads. As a corollary we get the smallest known example of an oriented tree (with 33 vertices) defining an NP-complete $\operatorname{CSP}(\mathbb{G})$.
\end{abstract}

\section{INTRODUCTION}

A digraph $\mathbb{G}$ is a finite set $V(\mathbb{G})$ of vertices with a set of edges $E(\mathbb{G}) \subseteq V(\mathbb{G})^{2}$. For digraphs $\mathbb{H}, \mathbb{G}$, a homomorphism $f: \mathbb{H} \rightarrow \mathbb{G}$ is an edge-preserving mapping $f: V(\mathbb{H}) \rightarrow V(\mathbb{G})$.

For a fixed finite digraph $\mathbb{G}, \operatorname{CSP}(\mathbb{G})$, also called the $\mathbb{G}$-coloring problem, is the following decision problem:

\section{INPUT: $\quad$ A digraph $\mathbb{H}$. \\ OUTPUT: Is there a homomorphism $\mathbb{H} \rightarrow \mathbb{G}$ ?}

This class of decision problems has received a great deal of attention lately, mainly because of the work of Feder and Vardi FV99. The authors conjectured a large natural class of NP problems avoiding the complexity classes between $\mathrm{P}$ and NP-complete (assuming $\mathrm{P} \neq \mathrm{NP}$ ). The class includes $k$-SAT problems, $k$-COLORING problems, solving a system of linear equations over finite fields and many others. In the same article they proved that each such problem is in the same

Received by the editors October 14, 2008, and, in revised form, January 5, 2009.

2000 Mathematics Subject Classification. Primary 05C85.

Key words and phrases. Graph coloring, constraint satisfaction problem, triad.

The first author was supported by the Grant Agency of the Czech Republic under grant Nos. 201/06/0664 and 201/09/P223, and by the project of the Ministry of Education under grant No. MSM 0021620839.

The second and fourth authors were supported by the Eduard Čech Center grant No. LC505.

The third author was partially supported by the Hungarian National Foundation for Scientific Research (OTKA), grant Nos. T 48809 and K 60148.

(C)2009 American Mathematical Society Reverts to public domain 28 years from publication 
complexity class as CSP $(\mathbb{G})$ for some digraph $\mathbb{G}$. Thus their dichotomy conjecture can be stated as follows:

Question 1.1. For every digraph $\mathbb{G}, \operatorname{CSP}(\mathbb{G})$ is either tractable or NP-complete.

For brevity, we say that $\mathbb{G}$ is NP-complete (tractable) if $\operatorname{CSP}(\mathbb{G})$ is NP-complete (tractable).

Dichotomy for undirected graphs was proved in HN90. For directed graphs, GWW92 verified that every oriented path is tractable. For oriented cycles, dichotomy was established in Fed01. A number of other cases were investigated and in some cases dichotomies proved; many are summarized in the book HN04]. Two important open cases were singled out: The first was a possible generalization of HN90 to digraphs without sources and sinks (i.e., digraphs such that every vertex has an incoming and outgoing edge), with an explicit dichotomy conjectured in BJH90]. The other was a quest to prove dichotomy for oriented trees (at the other end of the spectrum from digraphs without sources and sinks). Among trees, HNZ96a, HNZ96b focused on the simplest possible trees other than oriented paths, so-called triads, consisting of three oriented paths meeting at one vertex. Among the triads, the authors of HNZ96a, HNZ96b identified a subclass of so-called special triads, which have sufficient structure to allow at least some examples to be handled. The structure of special triads is examined in this paper.

The groundbreaking work of Jeavons, Cohen, and Gyssens [JCG97, successively developed and refined by Bulatov, Jeavons, and Krokhin [BJK05] and Larose and Tesson [LT07, has shown strong ties between the constraint satisfaction problem and universal algebra. This "algebraic approach" led to a rapid development of the subject; see the survey of Bulatov, Jeavons, Krokhin KBJ05 for an overview. Using a recent algebraic result of Maróti and McKenzie MM08, the authors BKN08a, BKN08b proved the dichotomy for digraphs with no sources or sinks, thus substantially generalizing the result of Hell and Nešetřil mentioned above and confirming the conjecture of BJH90].

The quest for dichotomy in trees remains elusive, but in this paper this is completely solved for special triads, proving dichotomy and giving a structural description of tractable and NP-complete triads. Such results seemed out of reach of non-algebraic methods. In particular, it seems the algebraic method yields definitely better tools to prove NP-completeness, as shown by a comparison of the proof of Theorem 3.4 and a proof in HNZ96a for one particular triad. The structural descriptions (of $\mathrm{P}$ and NP-complete cases) are given in terms of the existence of homomorphisms amongst the paths forming the special triads. These descriptions were first used in HNZ96b. There is an error in one of the cases considered there (Theorem 4.4 in HNZ96b), and this is fixed by the main result of the present paper (compatible mappings of HNZ96b] do not suffice to characterize easy instances; one has to refine this classification as done in Theorem 3.4).

As a byproduct we get a smallest known example of an NP-complete oriented tree; see Figure 1. The first such example was found in GWW92 (287 vertices), the construction was simplified in Gut91. (81 vertices), and a special triad with 45 vertices was found in [HNZ96a]. The special triad on Figure 1 has 33 vertices, and it seems quite likely that this is an NP-complete oriented tree with the smallest number of vertices, but we do not have a proof of this conjecture.

We wish to thank Pavol Hell and Jaroslav Nešetřil for their useful comments. 


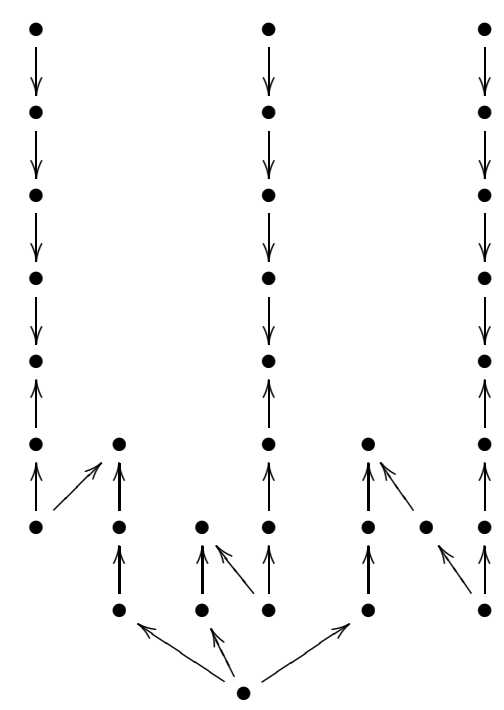

FiguRE 1. NP-complete special triad with minimal number of vertices (33)

\section{Digraphs, SPECIAL TRIAdS}

Let $\mathbb{G}$ be a digraph and $a, b \in V(\mathbb{G})$ its vertices. The fact that $(a, b) \in E(\mathbb{G})$ will be denoted by $a \stackrel{\mathbb{G}}{\longrightarrow} b$, or just $a \rightarrow b$ when $\mathbb{G}$ is clear from the context. Similarly we use $a \stackrel{\mathbb{G}}{\rightarrow} b$ and $a \nrightarrow b$ to denote that $(a, b) \notin E(\mathbb{G})$.

By $\mathbb{G}^{-1}$ we mean the digraph inverse to $\mathbb{G}$, that is, $V\left(\mathbb{G}^{-1}\right)=V(\mathbb{G})$ and $a \stackrel{\mathbb{G}^{-1}}{\longrightarrow} b$ iff $b \stackrel{\mathbb{G}}{\rightarrow} a$. By $\mathbb{G} \times \mathbb{H}$ we denote the direct product of the digraphs $\mathbb{G}$ and $\mathbb{H}$. If $\mathbb{G}, \mathbb{H}$ have the same set of vertices, we can form their composition $\mathbb{H} \circ \mathbb{G}$; that is, $V(\mathbb{H} \circ \mathbb{G})=V(\mathbb{G})$ and $a \stackrel{\mathbb{H} \circ \mathbb{G}}{\longrightarrow} b$ iff there exists $c$ such that $a \stackrel{\mathbb{G}}{\longrightarrow} c$ and $c \stackrel{\mathbb{H}}{\longrightarrow} b$. We say that a digraph $\mathbb{H}$ is a subdigraph of a digraph $\mathbb{G}$ if $V(\mathbb{H}) \subseteq V(\mathbb{G})$ and $E(\mathbb{H}) \subseteq E(\mathbb{G})$.

A digraph $\mathbb{G}$ is called a core if every homomorphism $\mathbb{G} \rightarrow \mathbb{G}$ is surjective. It is easy to see that for any digraph $\mathbb{G}$ we can find a core $\mathbb{G}^{\prime}$ such that

$$
\{\mathbb{H}: \mathbb{H} \rightarrow \mathbb{G}\}=\left\{\mathbb{H}: \mathbb{H} \rightarrow \mathbb{G}^{\prime}\right\} .
$$

In particular $\operatorname{CSP}(\mathbb{G})$ has the same complexity as $\operatorname{CSP}\left(\mathbb{G}^{\prime}\right)$.

An oriented tree is a digraph $\mathbb{G}$ which can be obtained from a tree $\mathbb{T}$ (undirected graph without a cycle) by orienting its edges; that is, for every edge $\{a, b\}$ of $\mathbb{T}$ we have either $a \stackrel{\mathbb{G}}{\longrightarrow} b$ or $b \stackrel{\mathbb{G}}{\longrightarrow} a$. For an oriented tree $\mathbb{G}$ we can find a mapping

$$
\text { level }: V(\mathbb{G}) \rightarrow\{0,1,2, \ldots\}
$$

such that level $(b)=\operatorname{level}(a)+1$ whenever $a \stackrel{\mathbb{G}}{\longrightarrow} b$. Clearly, there exists a unique such mapping with the smallest possible values. The value level $(a)$ for such a minimal mapping is called the level of the vertex $a$. The height hgt $(\mathbb{G})$ of $\mathbb{G}$ is the highest level of a vertex. 
An oriented path is a digraph obtained by orienting an undirected path. That is, an oriented path $\mathbb{P}$ has vertices $v_{0}, \ldots, v_{n}$ and edges $e_{0}, \ldots, e_{n-1}$, where $e_{i}$ is either $\left(v_{i}, v_{i+1}\right)$ or $\left(v_{i+1}, v_{i}\right)$. The net length of a path $\mathbb{P}$ is denoted by $\operatorname{alg}(\mathbb{P})$ and defined as

$$
\operatorname{alg}(\mathbb{P})=\left|\#\left\{i: v_{i} \stackrel{\mathbb{P}}{\rightarrow} v_{i+1}\right\}-\#\left\{i: v_{i+1} \stackrel{\mathbb{P}}{\rightarrow} v_{i}\right\}\right|
$$

$\mathbb{P}$ is called minimal if the net length of any of its subpaths is strictly smaller than $\operatorname{alg}(\mathbb{P})$. We can alternatively describe a minimal path as follows: a minimal path has a unique vertex of level 0 (called the initial vertex) and a unique vertex of level $\operatorname{alg}(\mathbb{P})$ (called the terminal vertex), and the initial and terminal vertices are the endpoints $\left\{v_{0}, v_{n}\right\}$ of the path. We will need the following easy lemma.

Lemma 2.1. Let $\mathbb{P}_{1}, \ldots, \mathbb{P}_{n}$ be minimal paths of the same net length $l$ with initial vertices $i_{1}, \ldots, i_{n}$ and terminal vertices $t_{1}, \ldots, t_{n}$, respectively. Let $\mathbb{Q}$ be an oriented tree of net length $l$. Then every homomorphism $f: \mathbb{P}_{1} \times \cdots \times \mathbb{P}_{n} \rightarrow \mathbb{Q}$ satisfies $\operatorname{level}\left(f\left(i_{1}, \ldots, i_{n}\right)\right)=0$ and level $\left(f\left(t_{1}, \ldots, t_{n}\right)\right)=l$.

Proof. It is not hard to prove (see [HHMNL88]) that there is a minimal path $\mathbb{S}$ of net length $l$ homomorphic to all of the paths $\mathbb{P}_{1}, \ldots, \mathbb{P}_{n}$. Let $a, b$ be the initial vertex and the terminal vertex of $\mathbb{S}$, respectively. Consider the natural homomorphism $g: \mathbb{S} \rightarrow \mathbb{P}_{1} \times \cdots \times \mathbb{P}_{n}$. Clearly $g(a)=\left\langle i_{1}, \ldots, i_{n}\right\rangle$ and $g(b)=\left\langle t_{1}, \ldots, t_{n}\right\rangle$. It can be readily seen that every homomorphism from a minimal path of net length $l$ to an oriented tree of net length $l$ maps the initial vertex to a vertex of level 0 and the terminal vertex to a vertex of level $l$. By using this fact for the homomorphism $f g$ : $\mathbb{S} \rightarrow \mathbb{Q}$ we obtain level $(f g(a))=0$ and level $(f g(b))=l$, and the claim follows.

A triad is an oriented tree with just one vertex of degree 3. We concentrate on a special case:

Definition 2.2. Let $\mathbb{P}_{1}, \mathbb{P}_{2}, \ldots, \mathbb{P}_{6}$ be minimal oriented paths of the same net length. By a special triad given by the paths $\mathbb{P}_{1}, \ldots, \mathbb{P}_{6}$ we mean the oriented tree obtained from the disjoint union of $\mathbb{P}_{1}, \ldots, \mathbb{P}_{6}$ by identifying the initial vertices of $\mathbb{P}_{1}, \mathbb{P}_{2}, \mathbb{P}_{3}$ into a single vertex 0 and identifying the terminal vertices of $\mathbb{P}_{i}$ and $\mathbb{P}_{i+3}$ to a single vertex $i$ for $i=1,2,3$.

A special triad is illustrated in Figure 2 (arrows on this picture denote "direction" of paths, not edges). A special triad has four vertices of level zero, namely $0,4,5,6$, and three vertices of the highest level, namely $1,2,3$. We assume, slightly abusing the formalism, that the paths $\mathbb{P}_{i}$ are subdigraphs of the special triad.

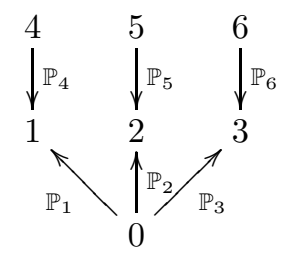

FiguRE 2. Special triad.

Note that a special triad defined in HNZ96b can be an inverse of our special triad (which, according to Definition [2.2, is not a special triad). All the results 
of our paper hold for such inverses, and our definition was chosen to simplify the presentation of the material.

\section{Compatible operations and CSP, main theorem}

By an $n$-ary operation on a set $A$ we understand a mapping $A^{n} \rightarrow A$, where $A^{n}=A \times \cdots \times A$ is the cartesian power of $A$. For a digraph $\mathbb{G}$ we say that an operation $w$ on $V(\mathbb{G})$ is compatible with $\mathbb{G}$ (or $w$ is compatible with $\mathbb{G}$ ) if $w$ is a homomorphism $w: \mathbb{G} \times \mathbb{G} \times \cdots \times \mathbb{G} \rightarrow \mathbb{G}$. In other words, $w$ is compatible with $\mathbb{G}$ if $a_{1} \stackrel{\mathbb{G}}{\longrightarrow} b_{1}, \ldots, a_{n} \stackrel{\mathbb{G}}{\longrightarrow} b_{n}$ imply $w\left(a_{1}, \ldots, a_{n}\right) \stackrel{\mathbb{G}}{\longrightarrow} w\left(b_{1}, \ldots, b_{n}\right)$.

It was shown in JCG97] that the complexity of $\operatorname{CSP}(\mathbb{G})$ depends only on the set of all compatible operations. In this article we use majority, totally symmetric idempotent and weak near-unanimity operations.

\section{Definition 3.1.}

- An $n$-ary operation $t$ on a set $A$ is said to be idempotent if

$$
t(a, a, \ldots, a)=a \quad \text { for every } a \in A .
$$

- A ternary operation $m$ on a set $A$ is called a majority operation if

$$
m(a, a, b)=m(a, b, a)=m(b, a, a)=a \quad \text { for any } a, b \in A .
$$

- An $n$-ary operation $t$ on a set $A$ is called totally symmetric if

$$
\left\{a_{1}, \ldots, a_{n}\right\}=\left\{b_{1}, \ldots, b_{n}\right\} \text { implies } t\left(a_{1}, \ldots, a_{n}\right)=t\left(b_{1}, \ldots, b_{n}\right)
$$

for any $a_{1}, \ldots, a_{n}, b_{1}, \ldots, b_{n} \in A$.

- An $n$-ary operation $w$ on a set $A$ is called a weak near-unanimity operation if it is idempotent and

$$
w(a, a, \ldots, a, b)=w(a, a, \ldots, a, b, a)=\cdots=w(b, a, a, \ldots, a)
$$

for any $a, b \in A$.

The existence of a compatible majority operation or a compatible totally symmetric idempotent operation of all arities ensures tractability [FV99]:

Theorem 3.2. Let $\mathbb{G}$ be a digraph satisfying one of the following conditions:

- $\mathbb{G}$ admits a compatible majority operation.

- For every $n \geq 1, \mathbb{G}$ admits a compatible $n$-ary totally symmetric idempotent operation.

Then $\operatorname{CSP}(\mathbb{G})$ is tractable.

On the other hand, non-existence of certain compatible operations implies NPcompleteness. The following theorem is a combination of the results in BJK05] and MM08.

Theorem 3.3. Let $\mathbb{G}$ be a digraph which is a core and admits no compatible weak near-unanimity operation. Then $\operatorname{CSP}(\mathbb{G})$ is NP-complete.

The algebraic dichotomy conjecture of Bulatov, Jeavons and Krokhin states (cf. BKJ00]) that the converse is also true; i.e. $\operatorname{CSP}(\mathbb{G})$ is conjectured to be tractable if $\mathbb{G}$ is a digraph admitting a compatible weak near-unanimity operation.

Now we can formulate our main theorem. 
Theorem 3.4. For every special triad $\mathbb{G}, \operatorname{CSP}(\mathbb{G})$ is either tractable or NPcomplete.

More specifically, let $\mathbb{G}$ be the special triad given by the paths $\mathbb{P}_{1}, \ldots, \mathbb{P}_{6}$.

(1) If there exist $i, j \in\{1,2,3\}, i \neq j$, and a homomorphism $\mathbb{P}_{i+3} \times \mathbb{P}_{j} \rightarrow \mathbb{P}_{i}$, then $\mathbb{G}$ admits a compatible totally symmetric idempotent operation of any arity.

(2) If there exist $i, j, k \in\{1,2,3\}$, pairwise distinct, and homomorphisms $\mathbb{P}_{i+3} \times$ $\mathbb{P}_{j+3} \times \mathbb{P}_{k} \rightarrow \mathbb{P}_{i}, \mathbb{P}_{i+3} \times \mathbb{P}_{j} \times \mathbb{P}_{k+3} \rightarrow \mathbb{P}_{i}, \mathbb{P}_{i+3} \times \mathbb{P}_{j} \times \mathbb{P}_{k} \rightarrow \mathbb{P}_{i}$, then $\mathbb{G}$ admits a compatible majority operation.

(3) Otherwise, $\operatorname{CSP}(\mathbb{G})$ is NP-complete.

It is easy to check that the special triad in Figure 1 satisfies neither (1) nor (2) (for instance $\mathbb{P}_{4} \times \mathbb{P}_{2} \nrightarrow \mathbb{P}_{1}$, since $\mathbb{P}_{2} \rightarrow \mathbb{P}_{4}$ and $\mathbb{P}_{2} \nrightarrow \mathbb{P}_{1}$ ). Moreover, among special triads which satisfy neither (1) nor (2), the one in Figure 1 has the minimal number of vertices.

\section{Tractable CASES}

In this section we describe all tractable special triads. In Section 5 we show that the remaining ones are NP-complete.

First we prove case (1) of Theorem 3.4

Lemma 4.1. Let $\mathbb{G}$ be the special triad given by the paths $\mathbb{P}_{1}, \ldots, \mathbb{P}_{6}$. Assume that there exist distinct $i, j \in\{1,2,3\}$ such that $\mathbb{P}_{i+3} \times \mathbb{P}_{j}$ is homomorphic to $\mathbb{P}_{i}$. Then $\mathbb{G}$ admits a compatible $n$-ary totally symmetric idempotent operation $t_{n}$ for every $n \geq 1$.

Proof. Let $\mathbb{H}$ be a graph with a vertex set $V(\mathbb{H})=\mathcal{P}(V(\mathbb{G})) \backslash\{\emptyset\}$ and such that

for $A, B \subseteq V(\mathbb{G})$ we have $A \stackrel{\mathbb{H}}{\longrightarrow} B$ if and only if for any $a \in A$ and $b \in B$ there exist $a^{\prime} \in A$ and $b^{\prime} \in B$ such that $a \stackrel{\mathbb{G}}{\longrightarrow} b^{\prime}$ and $a^{\prime} \stackrel{\mathbb{G}}{\longrightarrow} b$. It is easy to see (cf. DP99, Theorem 1]) that a homomorphism from $\mathbb{H}$ to $\mathbb{G}$ implies the existence of totally symmetric idempotent operations.

Without loss of generality we can assume that $i=2$ and $j=3$, so that we have a homomorphism

$$
f: \mathbb{P}_{5} \times \mathbb{P}_{3} \rightarrow \mathbb{P}_{2} .
$$

We define two auxiliary sets $U=V\left(\mathbb{P}_{3}\right) \backslash\{0,3\}$ consisting of inner vertices of the path $\mathbb{P}_{3}$ and $W=V(\mathbb{G}) \backslash U$. Moreover, we define a partial order on the set $V(\mathbb{G})$ according to the following schema: vertices appearing in a diagram below to the left are smaller (under $<$ ) than those appearing to the right:

$$
4 \stackrel{\mathbb{P}_{4}}{\longrightarrow} 1 \stackrel{\mathbb{P}_{1}}{\longleftarrow} \stackrel{\mathbb{P}_{2}}{\longrightarrow} 2 \stackrel{\mathbb{P}_{5}}{\longleftarrow} 5 \quad 3 \stackrel{\mathbb{P}_{6}}{\longleftarrow} 6
$$

and

$$
0 \longrightarrow \mathbb{P}_{3} \longrightarrow
$$

Note that the sets $U$ and $W$ are linearly ordered, and therefore the operation min is well defined on them.

The definition of a homomorphism $h$ from $\mathbb{H}$ to $\mathbb{G}$ is recursive: let $R \subseteq V(\mathbb{G})$.

(1) If not all the vertices from $R$ have the same level, then put $R^{\prime} \varsubsetneqq R$ to be the set of all the vertices of the lowest level in $R$; we define $h(R)$ to be equal to $h\left(R^{\prime}\right)$ otherwise. 
(2) If all the vertices from $R$ have the same level, then:

(2.1) if $R \cap U=\emptyset$, then we put $h(R)=\min (R \cap W)$;

(2.2) if $R \cap W=\emptyset$, then we put $h(R)=\min (R \cap U)$;

(2.3) if $R \cap U \neq \emptyset \neq R \cap W$, then

(2.3.1) if $\min (R \cap W)<2$, we put $h(R)=\min (R \cap W)$,

(2.3.2) if $2<\min (R \cap W)<5$, we put

$$
h(R)=f(\min (R \cap W), \min (R \cap U)) ;
$$

(2.3.3) if $5<\min (R \cap W)$, then we put $h(R)=\min (R \cap U)$.

Note that if any of the vertices $0, \ldots, 6$ belongs to $R$, then either case (11) or case (2.1) applies, so $h$ is well defined. It remains to prove that $h$ is a graph homomorphism.

Let us fix $R, S \in V(\mathbb{H})$ such that $R \stackrel{\mathbb{H}}{\longrightarrow} S$; we will show that $h(R) \stackrel{\mathbb{G}}{\longrightarrow} h(S)$. Let $R^{\prime}$ and $S^{\prime}$ be the vertices of the lowest level in $R$ and $S$ respectively. Obviously $R^{\prime} \stackrel{\mathbb{H}}{\rightarrow} S^{\prime}, h(R)=h\left(R^{\prime}\right), h(S)=h\left(S^{\prime}\right)$ and the homomorphism $h$ for $R^{\prime}$ and $S^{\prime}$ is defined in part (2). Let $l$ denote the level of vertices from $R^{\prime}$. Then the vertices in $S^{\prime}$ have level $l+1$.

It is easy to see that if $l \neq 0$ and $l+1 \neq \operatorname{hgt}(\mathbb{G})$, then the same subcase of (2) applies to the definitions of $h\left(R^{\prime}\right)$ and $h\left(S^{\prime}\right)$ and $h\left(R^{\prime}\right) \stackrel{\mathbb{G}}{\longrightarrow} h\left(S^{\prime}\right)$. Therefore we can assume that $l=0$ or $l+1=\operatorname{hgt}(\mathbb{G})$.

If $l=0$, then $R^{\prime} \subseteq\{0,4,5,6\}, R^{\prime} \cap U=\emptyset$ and $h\left(R^{\prime}\right)=\min \left(R^{\prime} \cap W\right)=$ $\min \left(R^{\prime}\right)$ (according to part (2.1) of the definition). Note that there exists a unique vertex of $\mathbb{G}$ which can belong to $U \cap S^{\prime}$; we denote this vertex by $s$. The proof splits into cases with respect to part of the definition for $h\left(S^{\prime}\right)$ :

- case (2.1): $\left(R^{\prime} \cap W\right)=R^{\prime} \stackrel{\mathbb{H}}{\longrightarrow} S^{\prime}=\left(S^{\prime} \cap W\right)$ and $h\left(R^{\prime}\right) \stackrel{\mathbb{G}}{\longrightarrow} h\left(S^{\prime}\right)$;

- case (2.3.1): $h\left(R^{\prime}\right) \in\{4,0\}$ and $h\left(S^{\prime}\right)$ is the unique vertex $s^{\prime}$ such that $h\left(R^{\prime}\right) \stackrel{\mathbb{P}_{4} \cup \mathbb{P}_{2}}{\longrightarrow} s^{\prime}$ and the case is solved;

- cases (2.2) and (2.3.3): $h\left(S^{\prime}\right)=s$, which implies that $0 \in R^{\prime} \subseteq\{0,6\}$ and further that $h\left(R^{\prime}\right)=0$; and finally

- case (2.3.2): $h\left(S^{\prime}\right)=f\left(s^{\prime}, s\right)$, where $s^{\prime}$ is such that $5 \stackrel{\mathbb{P}_{5}}{\longrightarrow} s^{\prime}$ and therefore $h\left(R^{\prime}\right)=0=f(5,0) \stackrel{\mathbb{G}}{\longrightarrow} f\left(s^{\prime}, s\right)=h\left(S^{\prime}\right)$, where the second equality follows from Lemma 2.1 .

In the other case $l+1=\operatorname{hgt}(\mathbb{G})$ and $S^{\prime}=S^{\prime} \cap W \subseteq\{1,2,3\}$. Therefore $h\left(S^{\prime}\right)$ is the minimal element of $S^{\prime}$. If $R^{\prime} \mp W$, then the conclusion is obvious, so we can assume that $3 \in S^{\prime}$ and $r \in R^{\prime} \cap U$ for the unique vertex $r$ such that $r \stackrel{\mathbb{P}_{3}}{\longrightarrow} 3$. As before, we consider cases with respect to the part of the definition for $h\left(R^{\prime}\right)$ :

- case (2.1) is impossible as $r \in R^{\prime} \cap U$;

- cases (2.2) and (2.3.3): $S^{\prime}=\{3\}$ and $h\left(R^{\prime}\right)=r$ and the conclusion holds;

- case (2.3.1): $\{1,2\} \cap S^{\prime} \neq \emptyset$ and $h\left(R^{\prime}\right) \stackrel{\mathbb{G}}{\rightarrow} \min \left(S^{\prime} \cap\{1,2\}\right)=h\left(S^{\prime}\right)$ as $h\left(S^{\prime}\right)<2$; and finally

- case (2.3.2): $h\left(R^{\prime}\right)=f\left(r^{\prime}, r\right)$, where $r^{\prime}$ is such that $r^{\prime} \stackrel{\mathbb{P}_{5}}{\longrightarrow} 2$ and therefore, using Lemma 2.1 $h\left(R^{\prime}\right)=f\left(r^{\prime}, r\right) \stackrel{\mathbb{G}}{\longrightarrow} f(2,3)=2=h\left(S^{\prime}\right)$.

This shows that $h$ is a graph homomorphism and concludes the proof.

Next we prove case (2) of Theorem 3.4 
Lemma 4.2. Let $\mathbb{G}$ be the special triad given by the paths $\mathbb{P}_{1}, \ldots, \mathbb{P}_{6}$. Let $i, j, k \in$ $\{1,2,3\}$ be pairwise different. Assume that there exist homomorphisms

$$
\mathbb{P}_{i+3} \times \mathbb{P}_{j+3} \times \mathbb{P}_{k} \rightarrow \mathbb{P}_{i}, \quad \mathbb{P}_{i+3} \times \mathbb{P}_{j} \times \mathbb{P}_{k+3} \rightarrow \mathbb{P}_{i}, \quad \mathbb{P}_{i+3} \times \mathbb{P}_{j} \times \mathbb{P}_{k} \rightarrow \mathbb{P}_{i}
$$

Then $\mathbb{G}$ admits a compatible majority operation.

Proof. Without loss of generality we can assume that $i=1, j=2, k=3$, so that we have homomorphisms

$$
u: \mathbb{P}_{4} \times \mathbb{P}_{5} \times \mathbb{P}_{3} \rightarrow \mathbb{P}_{1}, \quad v: \mathbb{P}_{4} \times \mathbb{P}_{2} \times \mathbb{P}_{6} \rightarrow \mathbb{P}_{1}, \quad w: \mathbb{P}_{4} \times \mathbb{P}_{2} \times \mathbb{P}_{3} \rightarrow \mathbb{P}_{1}
$$

Now we define the majority operation $m$ on $V(\mathbb{G})$. Let $a, b, c \in V(\mathbb{G})$.

(1) If $a, b, c$ have pairwise different levels, then we put $m(a, b, c)$ to be the vertex of the smallest level.

(2) If two of the vertices $a, b, c$ have the same level, say $k$, and the last one has a different level, then we put $m(a, b, c)=a$ if $a$ has level $k$, and $m(a, b, c)=b$ otherwise.

(3) If $a, b, c$ have the same level and lie on an oriented subpath of $\mathbb{G}$, then we put $m(a, b, c)$ to be the middle vertex from $a, b, c$ in this subpath.

(4) If $a, b, c$ have the same level and don't lie on an oriented path, then we can find a permutation $a^{\prime}, b^{\prime}, c^{\prime}$ of $a, b, c$ such that $a^{\prime}$ lies on $\mathbb{P}_{1}$ or $\mathbb{P}_{4}, b^{\prime}$ lies on $\mathbb{P}_{2}$ or $\mathbb{P}_{5}$, and $c^{\prime}$ lies on $\mathbb{P}_{3}$ or $\mathbb{P}_{6}$. Now
(a) If $a^{\prime} \in \mathbb{P}_{1}$, let $m(a, b, c)=a^{\prime}$.
(b) If $a^{\prime} \in \mathbb{P}_{4}, b^{\prime} \in \mathbb{P}_{2}, c^{\prime} \in \mathbb{P}_{3}$, let $m(a, b, c)=w\left(a^{\prime}, b^{\prime}, c^{\prime}\right)$.
(c) If $a^{\prime} \in \mathbb{P}_{4}, b^{\prime} \in \mathbb{P}_{2}, c^{\prime} \in \mathbb{P}_{6}$, let $m(a, b, c)=v\left(a^{\prime}, b^{\prime}, c^{\prime}\right)$.
(d) If $a^{\prime} \in \mathbb{P}_{4}, b^{\prime} \in \mathbb{P}_{5}, c^{\prime} \in \mathbb{P}_{3}$, let $m(a, b, c)=u\left(a^{\prime}, b^{\prime}, c^{\prime}\right)$.
(e) If $a^{\prime} \in \mathbb{P}_{4}, b^{\prime} \in \mathbb{P}_{5}, c^{\prime} \in \mathbb{P}_{6}$, let $m(a, b, c)=a^{\prime}$.

We claim that the definition of $m$ is correct. The only possibility where we can apply more rules is case 4 , where $a^{\prime}$ lies on both $\mathbb{P}_{1}$ and $\mathbb{P}_{4}$ or $b^{\prime}$ lies on both $\mathbb{P}_{2}$ and $\mathbb{P}_{5}$ or $c^{\prime}$ lies on both $\mathbb{P}_{3}$ and $\mathbb{P}_{6}$. But in this case, $a^{\prime}=1, b^{\prime}=2$ and $c^{\prime}=3$, and, by Lemma 2.1, the value is 1 in all the cases. It is also obvious that $m(a, a, b)=m(a, b, a)=m(b, a, a)=a$ (either case (2) or (3) applies).

It remains to show that $m$ is compatible with $\mathbb{G}$. Let $a, b, c, d, e, f \in V(\mathbb{G})$ be such that $a \rightarrow d, b \rightarrow e, c \rightarrow f$. We have to show that $m(a, b, c) \rightarrow m(d, e, f)$. As in the previous proof, it is easy to see that if the same cases apply for $m(a, b, c)$ and $m(d, e, f)$, then $m(a, b, c) \rightarrow m(d, e, f)$. The only remaining case is when the vertices $a, b, c$ have level 0 , lie on an oriented path and one of the cases $(b),(c),(d)$ applies for $m(d, e, f)$. It follows that $0 \in\{a, b, c\}$ and $m(a, b, c)=0$. Using Lemma2.1 we get $w(a, b, c)=v(a, b, c)=u(a, b, c)=0$. This implies $0 \rightarrow m(d, e, f)$.

Special triads which are not cores fall into case (1) of Theorem 3.4.

Lemma 4.3. Let $\mathbb{G}$ be a special triad which is not a core. Then there exist $i, j \in$ $\{1,2,3\}, i \neq j$ and a homomorphism $\mathbb{P}_{i+3} \times \mathbb{P}_{j} \rightarrow \mathbb{P}_{i}$.

Proof. Any homomorphism maps the set $\{4,5,6\}$ into $\{0,4,5,6\}$. If a homomorphism is an identity map on this set, then it is an identity map on $V(\mathbb{G})$ as well. In the opposite case there are distinct $i, j \in\{1,2,3\}$ such that either $\mathbb{P}_{i+3} \rightarrow \mathbb{P}_{i}$ or $\mathbb{P}_{j} \rightarrow \mathbb{P}_{i}$ (and $\left.\mathbb{P}_{j+3} \rightarrow \mathbb{P}_{i+3}\right)$. In both cases $\mathbb{P}_{i+3} \times \mathbb{P}_{j} \rightarrow \mathbb{P}_{i}$. 


\section{NP-COMPLETE CASES}

In this section we show that the special triads, which were not shown to be tractable in the previous section, are NP-complete.

So, let $\mathbb{G}$ be the special triad given by the paths $\mathbb{P}_{1}, \ldots, \mathbb{P}_{6}$ and assume that

(i) $\mathbb{G}$ is a core.

(ii) For all distinct $i, j \in\{1,2,3\}$ we have $\mathbb{P}_{i+3} \times \mathbb{P}_{j} \nrightarrow \mathbb{P}_{i}$.

(iii) For all pairwise distinct $i, j, k \in\{1,2,3\}$ we have $\mathbb{P}_{i+4} \times \mathbb{P}_{j+4} \times \mathbb{P}_{k} \nrightarrow \mathbb{P}_{i}$ or $\mathbb{P}_{i+4} \times \mathbb{P}_{j} \times \mathbb{P}_{k+4} \not \mathbb{P}_{i}$ or $\mathbb{P}_{i+4} \times \mathbb{P}_{j} \times \mathbb{P}_{k} \nrightarrow \mathbb{P}_{i}$

We will show that $\mathbb{G}$ has no compatible weak near-unanimity operation. This, together with Theorem 3.3. will conclude the proof of Theorem 3.4. Striving for a contradiction we assume that

(iv) $\mathbb{G}$ has a compatible $n$-ary weak near-unanimity operation $w$.

We will use the following construction.

Definition 5.1. Let $Z \subseteq\{1,2,3,4,5,6\}$ and let $i_{z}, t_{z}$ denote the initial and terminal vertices of $\mathbb{P}_{z}$, respectively (for all $z \in Z$ ). We define $\mathbb{G}^{Z}$ by

- $V\left(\mathbb{G}^{Z}\right)=V(\mathbb{G})$,

- $a \stackrel{\mathbb{G}^{Z}}{\longrightarrow} b$ if there exists a homomorphism $f: \prod_{z \in Z} \mathbb{P}_{z} \rightarrow \mathbb{G}$ such that $f\left(\left\langle i_{z}\right\rangle_{z \in Z}\right)=a$ and $f\left(\left\langle t_{z}\right\rangle_{z \in Z}\right)=b$.

From Lemma 2.1 it follows that $E\left(\mathbb{G}^{Z}\right) \subseteq\{(0,1),(0,2),(0,3),(4,1),(5,2),(6,3)\}$.

Next we show that the operation of taking the inverse digraph, the composition of digraphs and the construction in the last definition preserve compatible operations:

Lemma 5.2. Let $\mathbb{G}, \mathbb{H}$ be digraphs with the same vertex set, both compatible with an operation $t$. Then the graphs $\mathbb{G}^{-1}$ and $\mathbb{H} \circ \mathbb{G}$ are compatible with $t$. If $\mathbb{G}$ is a special triad and $Z \subseteq\{1,2,3,4,5,6\}$, then $\mathbb{G}^{Z}$ is compatible with $t$.

Proof. All of the constructions in the statement are special cases of primitive positive definitions, which are known (and easily seen) to preserve compatible operations.

Lemma 5.3. The set $\{1,2,3\}$ is closed under the operation $w$, i.e. $w(\bar{x}) \in\{1,2,3\}$ for every $\bar{x} \in\{1,2,3\}^{n}$.

Proof. Consider the digraph $\mathbb{H}=\mathbb{G}^{\{1,2,3,4,5,6\}}$ shown in Figure 3, Let $x_{1}, \ldots, x_{n} \in$

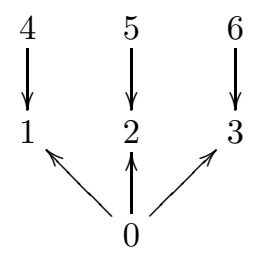

FIGURE 3. The digraph $\mathbb{G}^{\{1,2,3,4,5,6\}}$

$\{1,2,3\}$ be arbitrary. Since $0 \stackrel{\mathbb{H}}{\longrightarrow} 1,0 \stackrel{\mathbb{H}}{\longrightarrow} 2$ and $0 \stackrel{\mathbb{H}}{\longrightarrow} 3$ and $w$ is compatible with $\mathbb{H}$ (see the last lemma), we have $0=w(0,0, \ldots, 0) \stackrel{\mathbb{H}}{\longrightarrow} w\left(x_{1}, \ldots, x_{n}\right)$; therefore $w\left(x_{1}, \ldots, x_{n}\right) \in\{1,2,3\}$. 
We will use the previous claim in all that follows without explicit mentioning.

The assumptions (ii) and (iii) will be used to prove the existence of certain graphs compatible with $w$. Using (ii) we will construct $[a, b]$-graphs:

Definition 5.4. Let $a, b \in\{1,2,3\}$ be distinct. By an $[a, b]$-graph we mean any digraph $\mathbb{H}$ such that $V(\mathbb{H})=V(\mathbb{G})$ and

$$
a \stackrel{\mathbb{H}}{\rightarrow} a, b \stackrel{\mathbb{H}}{\longrightarrow} a, b \stackrel{\mathbb{H}}{\longrightarrow} b, b \stackrel{\mathbb{H}}{\longrightarrow} c, a \stackrel{\mathbb{H}}{\rightarrow} b, a \stackrel{\mathbb{H}}{\dagger} c,
$$

where $c$ is the element of $\{1,2,3\}$ distinct from $a$ and $b$.

An $[a, b]$-graph is depicted in Figure 4 (only vertices $1,2,3$ are drawn). In the picture, solid arrows are edges, dotted arrows mean that there is certainly no edge and no arrow means that there may be an edge or not.

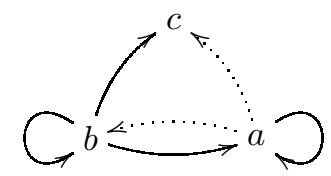

Figure 4. An $[a, b]$-graph.

Lemma 5.5. For any distinct $a, b \in\{1,2,3\}$, there exists an $[a, b]$-graph compatible with $w$.

Proof. Let us consider the case $a=1, b=2$; the other cases can be proved in the same way. From assumption (ii) we know that $\mathbb{P}_{4} \times \mathbb{P}_{2} \nrightarrow \mathbb{P}_{1}$; thus in the digraph $\mathbb{G}^{\{1,4\}}$ we have $4 \rightarrow 1,0 \rightarrow 2,0 \nrightarrow 1$ (see Figure 5 ). Now $\mathbb{G}^{\{1,2,3,4,5,6\}} \circ\left(\mathbb{G}^{\{1,4\}}\right)^{-1}$

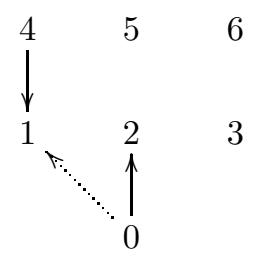

FIGURE 5. $\mathbb{G}^{\{1,4\}}$

is a [1,2]-graph. Lemma 5.2 tells us that this digraph is compatible with $w$.

Now we will use condition (iii) to obtain $[a, b, c]$-graphs:

Definition 5.6. Let $a, b, c \in\{1,2,3\}$ be pairwise distinct. By an $[a, b, c]$-graph we mean an $[a, b]$-graph with $c \rightarrow c$, i.e. any digraph $\mathbb{H}$ such that $V(\mathbb{H})=V(\mathbb{G})$ and

$$
a \stackrel{\mathbb{H}}{\rightarrow} a, b \stackrel{\mathbb{H}}{\longrightarrow} a, b \stackrel{\mathbb{H}}{\longrightarrow} b, b \stackrel{\mathbb{H}}{\longrightarrow} c, c \stackrel{\mathbb{H}}{\rightarrow} c, a \stackrel{\mathbb{H}}{\rightarrow} b, a \stackrel{\mathbb{H}}{\rightarrow} c .
$$

An $[a, b, c]$-graph is shown in Figure 6.

Lemma 5.7. For any distinct $a, b, c \in\{1,2,3\}$, there is an $[a, b, c]$-graph or an $[a, c, b]$-graph compatible with $w$. 


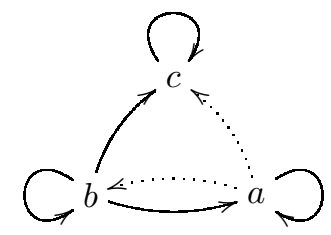

FiguRE 6. An $[a, b, c]$-graph.

Proof. The proof is similar to the proof of the previous lemma. Let us assume that $a=1, b=2, c=3$. From assumption (iii) we know that $\mathbb{P}_{4} \times \mathbb{P}_{5} \times \mathbb{P}_{3} \nrightarrow \mathbb{P}_{1}$ or $\mathbb{P}_{4} \times \mathbb{P}_{2} \times \mathbb{P}_{6} \nrightarrow \mathbb{P}_{1}$ or $\mathbb{P}_{4} \times \mathbb{P}_{2} \times \mathbb{P}_{3} \not \mathbb{P}_{1}$. In the first case we consider the digraph $\mathbb{K}=\mathbb{G}^{\{4,5,3\}}$, in the second case $\mathbb{K}=\mathbb{G}^{\{4,2,6\}}$ and in the third case $\mathbb{K}=\mathbb{G}^{\{4,2,3\}}$ (see Figure 7).
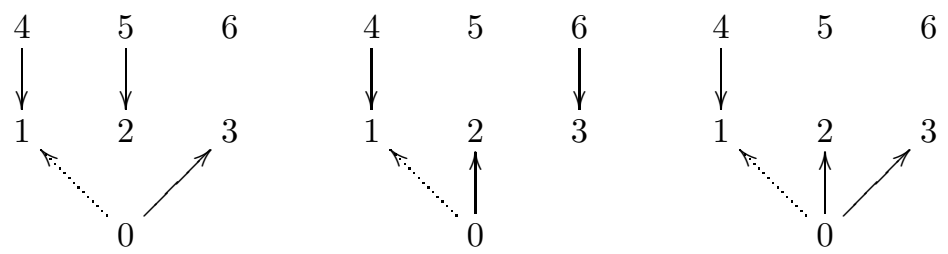

FiguRE 7. The graphs $\mathbb{K}$.

The digraph $\mathbb{H}=\mathbb{G}^{\{1,2,3,4,5,6\}} \circ \mathbb{K}^{-1}$ is either a $[1,2,3]$-graph (in the second and third cases) or a $[1,3,2]$-graph (in the first and third cases). From Lemma 5.2 we know that the operation $w$ is compatible with $\mathbb{H}$.

Lemma 5.8. For any $a, b \in\{1,2,3\}$ and tuple $\bar{x} \in\{a, b\}^{n}, t(\bar{x}) \in\{a, b\}$.

Proof. If $a=b$, then the claim follows from the idempotency of $w$, so assume that $a \neq b$. Let $c \in\{1,2,3\}$ be the element distinct from $a$ and $b$. Let $\mathbb{H}$ be either a $[c, a, b]$-graph or a $[c, b, a]$-graph, whose existence is provided by Lemma 5.7. If $\mathbb{H}$ is a $[c, b, a]$-graph, then $a \stackrel{\mathbb{H}}{\longrightarrow} a$ and $b \stackrel{\mathbb{H}}{\longrightarrow} a$, and from the compatibility of $w$, $w(\bar{x}) \stackrel{H}{\longrightarrow} w(a, a, \ldots, a)=a$. But now $w(\bar{x}) \in\{a, b\}$ as $c \stackrel{\mathbb{H}}{\rightarrow} a$. Similarly, if $\mathbb{H}$ is a $[c, a, b]$-graph, then $w(\bar{x}) \stackrel{\mathbb{H}}{\longrightarrow} b$, and we get the same conclusion.

Lemma 5.9. If $w(a, b, \ldots, b)=a$ for a pair $a, b \in\{1,2,3\}$ of distinct elements, then $t(a, \bar{x})=a$ for all $\bar{x} \in\{1,2,3\}^{n-1}$.

Proof. Let $\mathbb{H}$ be an $[a, b]$-graph from Lemma 5.5. Then $a \stackrel{\mathbb{H}}{\rightarrow} a, b \stackrel{\mathbb{H}}{\rightarrow} a, b \stackrel{\mathbb{H}}{\rightarrow} b$ and $b \stackrel{\mathbb{H}}{\longrightarrow} c$; therefore $a=w(a, b, \ldots, b) \stackrel{\mathbb{H}}{\longrightarrow} w(a, \bar{x})$. However, the only edge from $a$ to the set $\{1,2,3\}$ is $a \stackrel{\mathbb{H}}{\longrightarrow} a$, which proves that $w(a, \bar{x})=a$.

Lemma 5.10. $w(a, b, \ldots, b)=b$ for any $a, b \in\{1,2,3\}$.

Proof. Suppose that $w(a, b, \ldots, b) \neq b$ for some $a, b \in\{1,2,3\}$. Then $w(a, b, \ldots, b)$ $=a$ by Lemma 5.8. Let $c$ be the element of $\{1,2,3\}$ distinct from $a, b$. From Lemma 5.9 we know that $w(a, c, \ldots, c)=a$. 
Let $\mathbb{H}$ be a $\langle b, c\rangle$-graph. Since $c \stackrel{\mathbb{H}}{\longrightarrow} a$ and $b \stackrel{\mathbb{H}}{\longrightarrow} b$, we have $w(c, b, \ldots, b) \stackrel{H}{\longrightarrow}$ $w(a, b, \ldots, b)=a$. Therefore $w(c, b \ldots, b) \neq b$. From Lemma 5.8 it follows that $w(c, b, \ldots, b)=c$.

As $w$ is a weak near-unanimity operation we also have $w(b, \ldots, b, c)=c$, and by applying Lemma 5.9 with permuted variables we get that $w(\bar{x}, c)=c$ for any $\bar{x} \in\{1,2,3\}^{n-1}$. In particular, $w(a, c, \ldots, c)=c$, a contradiction.

Lemma 5.11. $w(a, b, c, \ldots, c)=c$ for any pairwise distinct elements $a, b, c \in$ $\{1,2,3\}$.

Proof. Suppose $w(a, b, c, \ldots, c)=a$. Let $\mathbb{H}$ be either an $[a, b, c]$ or an $[a, c, b]$-graph compatible with $w$.

If $\mathbb{H}$ is an $[a, b, c]$-graph, then $a \stackrel{\mathbb{H}}{\longrightarrow} a, b \stackrel{\mathbb{H}}{\longrightarrow} c$ and $c \stackrel{\mathbb{H}}{\longrightarrow} c$, so $a=w(a, b, c, \ldots, c) \stackrel{\mathbb{H}}{\longrightarrow}$ $w(a, c, \ldots, c)=c$, where the last equality follows from Lemma 5.10, a contradiction.

If $\mathbb{H}$ is an $[a, c, b]$-graph, we get $a=w(a, b, c, \ldots, c) \stackrel{\mathbb{H}}{\longrightarrow} w(a, b, \ldots, b)=b$, which is again a contradiction.

The fact that $w(a, b, c, \ldots, c) \neq b$ can be proved analogously.

Lemma 5.12. $w(a, a, c, \ldots, c)=c$ for all $a, c \in\{1,2,3\}$.

Proof. We can clearly assume that $a \neq c$ and let $b \in\{1,2,3\} \backslash\{a, c\}$. From the last claim we know that $t(a, b, c, \ldots, c)=c$.

Let $\mathbb{H}$ be a $[c, a, b]$ - or $[c, b, a]$-graph compatible with $w$. We will show the reasoning for $\mathbb{H}$ being a $[c, a, b]$-graph; the second possibility is analogous. Since $a \stackrel{\mathbb{H}}{\longrightarrow} b, b \stackrel{\mathbb{H}}{\longrightarrow} b, c \stackrel{\mathbb{H}}{\longrightarrow} c$, we have $c=w(a, b, c, \ldots, c) \stackrel{\mathbb{H}}{\longrightarrow} w(b, b, c, \ldots, c)$ and therefore $w(b, b, c, \ldots, c)=c$. Let $\mathbb{K}$ be a $[c, b]$-graph compatible with $w$. We get $c=w(b, b, c, \ldots, c) \stackrel{\mathbb{K}}{\longrightarrow} w(a, a, c, \ldots, c)$; thus $w(a, a, c, \ldots, c)=c$.

By repeating the arguments of the last two lemmas we obtain $w(a, a, b, c, \ldots, c)=$ $c$ and $w(a, a, a, c, \ldots, c)=c$ after the first repetition, $w(a, a, a, b, c, \ldots, c)=c$ and $w(a, a, a, a, c, \ldots, c)=c$ after the second and so on until $w(a, \ldots, a, c)=c$. But $w(a, \ldots, a, c)=w(c, a, \ldots, a)=a$ by Lemma 5.10 which is a contradiction. This finishes the proof of the NP-complete case and Theorem 3.4 .

\section{ADDED AFTER POSTING}

This section corrects two mistakes in the article. The first mistake concerns the situation when a special triad is not a core: the characterization given in Lemma 4.3 is incomplete, as it can happen that all the paths $\mathbb{P}_{1}, \ldots, \mathbb{P}_{6}$ are mapped to one of the paths $\mathbb{P}_{4}, \ldots, \mathbb{P}_{6}$. Unfortunately this is the case in the example of the special triad in Figure 1. To obtain a special triad which is NP-complete, we replace the path $\mathbb{P}_{4}$ by $\mathbb{P}_{2}$ and paths $\mathbb{P}_{5}, \mathbb{P}_{6}$ both by $\mathbb{P}_{1}$ (the new triad has 39 vertices). This mistake doesn't influence the dichotomy result for special triads, as it is well known that $\operatorname{CSP}(\mathbb{G})$ is tractable for any oriented path $\mathbb{G}$.

The second mistake is in the classification given in Theorem 3.4. To correct it we need an auxiliary notation: for oriented paths $\mathbb{P}_{1}, \ldots, \mathbb{P}_{k}$ with initial vertices $i_{1}, \ldots, i_{k}$ let $c\left(\mathbb{P}_{1} \times \cdots \times \mathbb{P}_{k}\right)$ be the connectivity component of the digraph $\mathbb{P}_{1} \times$ $\cdots \times \mathbb{P}_{k}$ containing the vertex $\left(i_{1}, \ldots, i_{k}\right)$. The right statement of Theorem 3.4 is obtained by replacing all products with their connectivity components: 
Theorem 3.4. For every special triad $\mathbb{G}, \operatorname{CSP}(\mathbb{G})$ is either tractable or NPcomplete.

More specifically, let $\mathbb{G}$ be the special triad given by paths $\mathbb{P}_{1}, \ldots, \mathbb{P}_{6}$.

(1) If there exist $i, j \in\{1,2,3\}, i \neq j$, and a homomorphism $c\left(\mathbb{P}_{i+3} \times \mathbb{P}_{j}\right) \rightarrow \mathbb{P}_{i}$, then $\mathbb{G}$ admits a compatible totally symmetric idempotent operation of any arity.

(2) If there exist $i, j, k \in\{1,2,3\}$ pairwise distinct and homomorphisms $c\left(\mathbb{P}_{i+3} \times \mathbb{P}_{j+3} \times \mathbb{P}_{k}\right) \rightarrow \mathbb{P}_{i}, c\left(\mathbb{P}_{i+3} \times \mathbb{P}_{j} \times \mathbb{P}_{k+3}\right) \rightarrow \mathbb{P}_{i}, c\left(\mathbb{P}_{i+3} \times \mathbb{P}_{j} \times \mathbb{P}_{k}\right) \rightarrow \mathbb{P}_{i}$, then $\mathbb{G}$ admits a compatible majority operation.

(3) If $\mathbb{G}$ is not a core, then either one of the cases (1), (2) can be applied, or the core of $\mathbb{G}$ is an oriented path.

(4) Otherwise, $\operatorname{CSP}(\mathbb{G})$ is NP-complete.

The proof of the theorem remains the same except for replacing all products of oriented paths with their connectivity component containing the initial vertex. This change is necessary because in their original formulation Lemmas 5.5 and 5.7 might not be true - for example in Lemma 5.5 the nonexistence of a homomorphism from $\mathbb{P}_{4} \times \mathbb{P}_{2}$ to $\mathbb{P}_{1}$ doesn't necessarily imply that $0 \nrightarrow 1$ in $\mathbb{G}^{\{2,4\}}$, since a homomorphism can map the other connectivity components of $\mathbb{P}_{4} \times \mathbb{P}_{2}$ outside the path $\mathbb{P}_{1}$.

Because of this change we also need to adjust the proofs of Lemmas 4.1 and 4.2 , In Lemma 4.1 when defining the homomorphism $h$ from $\mathbb{H}$ to $\mathbb{G}$ we need to distinguish one more case:

(0) If all the vertices in $R$ have the same level and are in a connectivity component of $\mathbb{H}$ other than $\{0\}$, then we put $h(R)$ to be the smallest vertex in $R$ in the ordering

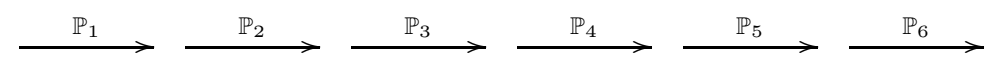

Note that in this case $R \cap\{0,1,2,3,4,5,6\}=\emptyset$.

In the proof of Lemma 4.2 we also need to add one more case:

(0) If $a, b, c$ have the same level, don't lie on an oriented subpath of $\mathbb{G}$ and $(a, b, c)$ is in a connectivity component of $\mathbb{G}^{3}$ other than the vertex $(0,0,0)$, then we put $m(a, b, c)=a$.

We wish to thank Jakub Bulín for carefully reading the article and finding the above two mistakes.

\section{REFERENCES}

[BJH90] Jørgen Bang-Jensen and Pavol Hell, The effect of two cycles on the complexity of colourings by directed graphs, Discrete Appl. Math. 26 (1990), no. 1, 1-23. MR:1028872 (91c:05072)

[BJK05] Andrei Bulatov, Peter Jeavons, and Andrei Krokhin, Classifying the complexity of constraints using finite algebras, SIAM J. Comput. 34 (2005), no. 3, 720-742 (electronic). MR2137072 (2005k:68181)

[BKJ00] Andrei A. Bulatov, Andrei A. Krokhin, and Peter Jeavons, Constraint satisfaction problems and finite algebras, Automata, languages and programming (Geneva, 2000), Lecture Notes in Comput. Sci., vol. 1853, Springer, Berlin, 2000, pp. 272-282. MR:1795899 (2001h:68137)

[BKN08a] Libor Barto, Marcin Kozik, and Todd Niven, The CSP dichotomy holds for digraphs with no sources and no sinks (a positive answer to a conjecture of Bang-Jensen and Hell), SIAM Journal on Computing 38 (2009), no. 5, 1782-1802. 
[BKN08b] - Polymorphisms and the complexity of homomorphism problems, Proceedings of the 40th ACM Symposium on Theory of Computing, STOC'08, 2008.

[DP99] Victor Dalmau and Justin Pearson, Closure functions and width 1 problems, Fifth International Conference on Principles and Practice of Constraint Programming (CP'99), 1999, Lecture Notes in Computer Science, vol. 1713, Springer-Verlag, 2004, pp. 159-173.

[Fed01] Tomás Feder, Classification of homomorphisms to oriented cycles and of k-partite satisfiability, SIAM J. Discrete Math. 14 (2001), no. 4, 471-480 (electronic). MR.1861785 (2002j:05063)

[FV99] Tomás Feder and Moshe Y. Vardi, The computational structure of monotone monadic SNP and constraint satisfaction: A study through Datalog and group theory, SIAM J. Comput. 28 (1999), no. 1, 57-104 (electronic). MR1630445 (2000e:68063)

[Gut91] W. Gutjahr, Graph colourings, Ph.D. Thesis, Free University Berlin, 1991.

[GWW92] Wolfgang Gutjahr, Emo Welzl, and Gerhard Woeginger, Polynomial graph-colorings, Discrete Appl. Math. 35 (1992), no. 1, 29-45. MR.1138082 (92m:05081)

[HHMNL88] R. Häggkvist, P. Hell, D. J. Miller, and V. Neumann Lara, On multiplicative graphs and the product conjecture, Combinatorica 8 (1988), no. 1, 63-74. MR.951994 (90e:05037)

[HN90] Pavol Hell and Jaroslav Nešetřil, On the complexity of H-coloring, J. Combin. Theory Ser. B 48 (1990), no. 1, 92-110. MR.1047555 (91m:68082)

[HN04] _ Graphs and homomorphisms, Oxford Lecture Series in Mathematics and its Applications, vol. 28, Oxford University Press, Oxford, 2004. MR2089014 (2005k:05002)

[HNZ96a] P. Hell, J. Nešetřil, and X. Zhu, Complexity of tree homomorphisms, Discrete Appl. Math. 70 (1996), no. 1, 23-36. MR1402736 (97e:68049)

[HNZ96b] Duality and polynomial testing of tree homomorphisms, Trans. Amer. Math. Soc. 348 (1996), no. 4, 1281-1297. MR.1333391 (96h:05072)

[JCG97] Peter Jeavons, David Cohen, and Marc Gyssens, Closure properties of constraints, J. ACM 44 (1997), no. 4, 527-548. MR1481313(99a:68089)

[KBJ05] Andrei Krokhin, Andrei Bulatov, and Peter Jeavons, The complexity of constraint satisfaction: An algebraic approach, Structural theory of automata, semigroups, and universal algebra, NATO Sci. Ser. II Math. Phys. Chem., vol. 207, Springer, Dordrecht, 2005; notes taken by Alexander Semigrodskikh, pp. 181-213. MR2210131 (2006m:68053)

[LT07] Benoit Larose and Pascal Tesson, Universal algebra and hardness results for constraint satisfaction problems, ICALP (2007), Lecture Notes in Comput. Sci., 4596, Springer, Berlin, 2007. MR2424689

[MM08] Miklós Maróti and Ralph McKenzie, Existence theorems for weakly symmetric operations, Algebra Universalis 59 (2008), no. 3-4, 463-489.

Department of Algebra, Charles University, Prague, Czech Republic

E-mail address: libor.barto@gmail.com

Department of Theoretical Computer Science, Jagiellonian University, Krakow, Poland - and - Eduard Čech Center, Prague, Czech Republic

E-mail address: kozik@tcs.uj.edu.pl

Bolyai Institute, University of Szeged, Szeged, Hungary

E-mail address: mmaroti@math.u-szeged.hu

Eduard Čech Center, Prague, Czech Republic

E-mail address: niven@karlin.mff.cuni.cz 